\title{
Immunometabolites Drive Bacterial Adaptation to the Airway
}

\author{
Kira L. Tomlinson, Alice S. Prince and Tania Wong Fok Lung* \\ Department of Pediatrics, Vagelos College of Physicians \& Surgeons, Columbia University, New York, NY, United States
}

\section{OPEN ACCESS}

Edited by:

Tammy Kielian,

University of Nebraska Medical Center,

United States

Reviewed by:

Daniel Wozniak,

The Ohio State University,

United States

Werner Solbach,

University of Lübeck, Germany

*Correspondence:

Tania Wong Fok Lung

tw2595@cumc.columbia.edu

Specialty section:

This article was submitted to

Microbial Immunology,

a section of the journal

Frontiers in Immunology

Received: 06 October 2021 Accepted: 08 November 2021

Published: 25 November 2021

Citation:

Tomlinson KL, Prince AS and

Wong Fok Lung T (2021)

Immunometabolites Drive Bacterial

Adaptation to the Airway.

Front. Immunol. 12:790574.

doi: 10.3389/fimmu.2021.790574
Pseudomonas aeruginosa and Staphylococcus aureus are both opportunistic pathogens that are frequently associated with chronic lung infections. While bacterial virulence determinants are critical in initiating infection, the metabolic flexibility of these bacteria promotes their persistence in the airway. Upon infection, these pathogens induce host immunometabolic reprogramming, resulting in an airway milieu replete with immunesignaling metabolites. These metabolites are often toxic to the bacteria and create a steep selection pressure for the emergence of bacterial isolates adapted for long-term survival in the inflamed lung. In this review, we discuss the main differences in the host immunometabolic response to $P$. aeruginosa and $S$. aureus, as well as how these pathogens alter their own metabolism to adapt to airway metabolites and cause persistent lung infections.

Keywords: immunometabolism, host-pathogen interaction, bacterial persistence, metabolic adaptation, itaconate, succinate, Staphylococcus aureus, Pseudomonas aeruginosa

\section{INTRODUCTION}

Healthcare-associated pneumonias, including those associated with COVID-19, are caused by opportunistic pathogens that readily adapt to the human airway. While the host immune response to lung pathogens has been extensively characterized $(1,2)$, less is known about how opportunistic bacteria survive in the lung despite hostile inflammatory conditions and appropriate antibiotic treatment.

Bacterial infection of the airway occurs in stages. Initially, environmental pathogens withstand the host immune response and antibiotic treatment by using virulence factors and acquired antimicrobial resistance genes to establish infection. Once a nidus of infection is formed, bacteria alter their metabolism and selectively regulate virulence to promote survival in the limited nutrient conditions and oxidative environment of the airway (3). Traditional experiments in microbial pathogenesis, including the use of defined deletion mutants and complemented strains, have been very effective in defining the virulence factors that are critical for establishing acute pneumonia (4, 5). However, the adaptations that enable bacteria to persist in the airway are not well understood.

To study adaptation of bacteria to the human airway, multiple research groups have used clinical strains from patients with chronic or persistent pneumonias, such as those with Cystic Fibrosis (CF) (6-8). The natural history of pulmonary infection in CF typically consists of initial infection with Staphylococcus aureus, followed by years of S. aureus and Pseudomonas aeruginosa co-infection, then predominant and intractable $P$. aeruginosa infection coinciding with increased pulmonary exacerbations and declining lung function (9). S. aureus and $P$. aeruginosa strains can thus be isolated from the same CF patient over years $(10,11)$. Genomic, transcriptional, and phenotypic 
data from these longitudinal isolates reveal the strategies that enable these common Gram-positive and Gram-negative pathogens to adapt to the airway for long-term survival. While the CF airway is usually polymicrobial, bacterial pneumonia, especially when attributed to the antibiotic-resistant ESKAPE pathogens, is often considered a single entity. This review will discuss the different strategies that $P$. aeruginosa and $S$. aureus use to survive in the human airway as well as the host factors that drive bacterial adaptation, with a particular focus on the roles of both host and bacterial metabolism.

\section{P. AERUGINOSA INDUCES A SUCCINATE- DOMINATED HOST METABOLIC REPROGRAMMING}

The pathogenesis of acute $P$. aeruginosa infection often involves lipopolysaccharide (LPS)-displaying bacteria that activate host pattern recognition receptors (PRRs) like the Toll-like receptors (TLRs) (12). This bacterial recognition by host cells leads to downstream proinflammatory cytokine expression and phagocytic recruitment for bacterial clearance $(13,14)$. Recently, it has become increasingly appreciated that this inflammatory response is driven by changes in the metabolic activity of immune cells, a process called immunometabolism.

In response to LPS, macrophages become activated through TLR4 signaling and undergo metabolic reprogramming (15). This comprises upregulation of aerobic glycolysis and downregulation of oxidative phosphorylation (OXPHOS) to meet the cell's energy requirements (Figure 1). While this metabolic shift may seem counterintuitive given that OXPHOS is more energy efficient (36 molecules of ATP/glucose molecule), aerobic glycolysis can generate ATP (2 molecules of ATP/glucose molecule) faster than OXPHOS, akin to the Warburg effect in cancer cells $(15,16)$. These metabolic changes are accompanied by increased production of the metabolite succinate through glutamine-dependent anaplerosis and the gamma-aminobutyric acid (GABA)-shunt (17) (Figure 1). Increased mitochondrial oxidation of succinate to fumarate via succinate dehydrogenase (SDH/respiratory complex II) and the resulting elevation of mitochondrial membrane potential $(\Psi)$ drive the production of reactive oxygen species (ROS) via reverse electron transport (RET) (18) (Figure 1). Succinate and ROS stabilize the host transcription factor hypoxia-inducible factor $1 \alpha$ (HIF-1 $\alpha$ ) by inhibition of prolyl hydroxylase (PHD) in the cytosol (19) (Figure 1). This causes an increase in the transcription of genes encoding glycolytic enzymes and the proinflammatory cytokine IL$1 \beta$, which is expected to promote bacterial clearance.

It is important to note that, in addition to fueling succinate production, glutamine-dependent anaplerosis can also increase $\alpha$ ketoglutarate levels. $\alpha$-ketoglutarate promotes anti-inflammatory pathways through epigenetic changes [reviewed in (20)]. Therefore, glutaminolysis can drive both pro-inflammatory and antiinflammatory metabolic programs, and the ratio of succinate to $\alpha$ ketoglutarate is critical for determining macrophage polarization (21).

While these metabolic changes have been extensively detailed in macrophages responding to LPS in vitro, infection with live
$P$. aeruginosa also results in increased succinate accumulation and production of IL-1 $\beta$ in the murine lung (22). Of note, increased succinate production is an inherent property of the CF lung, even in the absence of infection (22), due to a lack of sufficient membranebound CF transmembrane conductance regulator (CFTR) and impaired activity of the metabolic regulator Phosphatase and Tensin Homolog deleted on Chromosome 10 (PTEN) (22, 23).

Increases in proinflammatory cytokines do not always clear pathogens. This is clearly exemplified by the current COVID-19 pandemic, whereby SARS-CoV2-induced cytokine storm results in excessive inflammation that fails to clear the viral pathogen and instead contributes to immunopathology and mortality (24, 25). $P$. aeruginosa also stimulates IL- $1 \beta$ production by activating the NLRC4 inflammasome in alveolar macrophages, which enhances bacterial infection in a murine pneumonia model (26). Dampening inflammasome activation or inhibiting IL-1 $\beta$ signaling via the use of $\mathrm{Il}-1 \mathrm{r}$ or $\mathrm{Il}-18 \mathrm{r}$ null mice promotes bacterial clearance and reduces immunopathology (26).

In order to restore homeostatic balance and counteract succinatedriven inflammation, myeloid cells upregulate the expression of Immune Responsive Gene 1 (Irg1/Acod1) to produce the immunometabolite itaconate (Figure 1). Interestingly, this dicarboxylate, which structurally resembles succinate, dampens inflammation via its effect on several host pathways described below. Itaconate is abundantly produced in the host airway during $P$. aeruginosa infection as well as in the CF airway $(22,27)$.

\section{ITACONATE COUNTERACTS SUCCINATE- DRIVEN INFLAMMATION}

Although itaconate was originally discovered in 1836 (28), its immunoregulatory function was only elucidated in $2016(29,30)$. Using Irg1 knockout cells and exogenous itaconate, multiple groups found that itaconate inhibited $\mathrm{SDH}$, thus preventing succinate oxidation, ROS production via RET, HIF- $1 \alpha$ stabilization, and IL$1 \beta$ production (29-31) (Table 1). Further investigations have utilized derivatives like 4-octyl-itaconate (4OI) and dimethylitaconate (DMI) to show that itaconate mitigates inflammation by modifying cysteine residues, inhibiting glycolytic enzymes that energetically sustain immune cell activation, preventing NLRP3 inflammasome activation, and activating anti-inflammatory and anti-oxidant pathways by promoting ATF3 and NRF2 activation $(32,33,36-38,40,41)$ (Table 1). These pathways subsequently drive macrophage polarization. For example, itaconate plays a key role in IL-33-induced alternatively activated macrophages (AAMs) by promoting GATA3 expression (42). Itaconate and its derivatives remain the focus of ongoing investigation, even as a potential therapeutic agent to treat COVID-19 (43), given their role in the control of other viruses such as Zika (44) and influenza (45).

Recently, some of the mechanisms of action initially ascribed to itaconate are being reevaluated in light of major differences in the effects of itaconate versus its derivatives (34) (Table 1). DMI and 4OI were originally used because of their cell permeability, but multiple studies have shown that itaconate also reaches the cytoplasm when administered exogenously to cells $(34,37)$. 


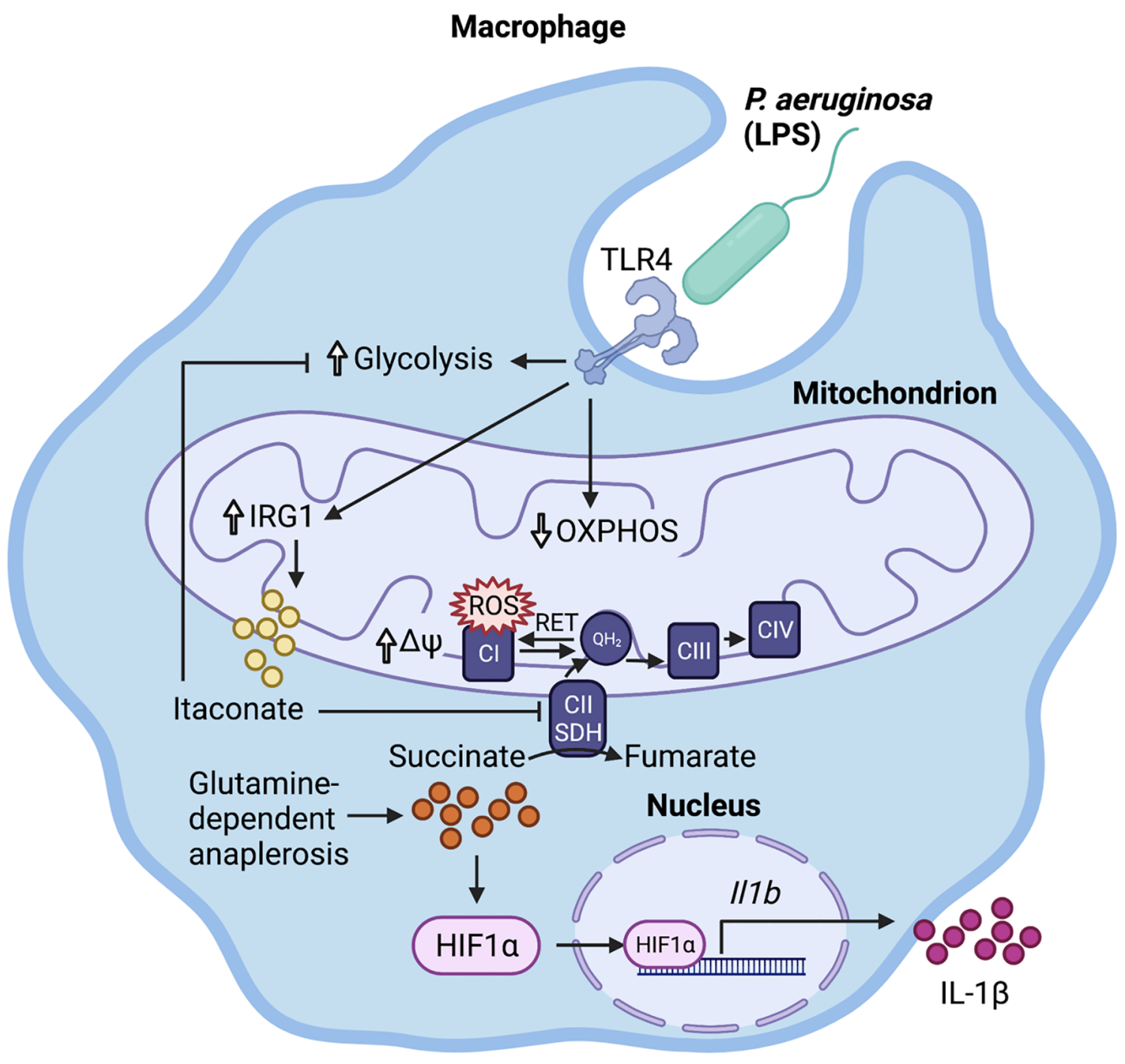

FIGURE 1 | Immunometabolic response to $P$. aeruginosa. LPS activates the infected macrophage, stimulating glycolysis and downregulating oxidative phosphorylation (OXPHOS). This promotes glutamine-dependent anaplerosis, which replenishes succinate for oxidation to fumarate by mitochondrial succinate dehydrogenase (SDH). The resulting increase in mitochondrial transmembrane potential $(\Delta \Psi)$ and over-reduction of the ubiquinone pool reverse electron flow to complex I, where they escape as reactive oxygen species (ROS). Both ROS and succinate stabilize HIF-1 $\alpha$, which translocates to the nucleus and binds to its target promoter regions, increasing $/ /-$ $1 \beta$ transcription. The succinate-driven inflammation is regulated by the production of the anti-inflammatory metabolite itaconate.

Direct comparisons of itaconate and its derivatives have revealed that DMI and 4OI are more electrophilic than itaconate and, subsequently, target different processes within mammalian cells. While DMI and 4OI are able to activate ATF3 and NRF2 signaling, itaconate does not (34) (Table 1). Additionally, itaconate promotes Type I interferon signaling, while its derivatives suppress IFN- $\beta$ production (34) (Table 1). Despite these differences, itaconate and its derivatives share some core mechanisms of action, including inhibition of glycolysis, inhibition of succinate oxidation, suppression of pro-inflammatory cytokine release, inhibition of the NLRP3 inflammasome, and promotion of ROS generation through OXPHOS and the pentose phosphate pathway (PPP) (Table 1). Overall, itaconate regulates inflammation by modulating the metabolic reprogramming that enables immune cells to release cytokines and antibacterial factors into the surrounding tissue.

\section{SUCCINATE AND ITACONATE FUEL $P$. AERUGINOSA LUNG INFECTION}

As host cells undergo immunometabolic reprogramming and release metabolites, cytokines, and antimicrobial factors into the lung environment, $P$. aeruginosa adapts through its own metabolic flexibility. This metabolic versatility is conferred by a global regulatory system termed carbon catabolite repression (CCR) that 
TABLE 1 | Mechanisms of action of itaconate, and its derivatives.

\begin{tabular}{|c|c|c|c|c|c|c|c|}
\hline Confirmed & Contested & Immune responsive gene1 (Irg1) & Itaconate & \multicolumn{2}{|c|}{ 4-Octyl-Itaconate } & Dimethy & conate \\
\hline \multicolumn{2}{|c|}{$\begin{array}{l}\text { Modifies cysteine residues } \\
\text { and inhibits metabolic } \\
\text { enzymes }\end{array}$} & & (31) & \multicolumn{2}{|c|}{$(32,33)$} & & \\
\hline \multicolumn{2}{|c|}{$\begin{array}{l}\text { Inhibits SDH and induces } \\
\text { succinate accumulation }\end{array}$} & $(29,30,34)$ & $(29,30,34)$ & \multicolumn{2}{|c|}{ (34) } & (30) & (34) \\
\hline \multicolumn{2}{|c|}{$\begin{array}{l}\text { Suppresses IL-1 } \beta, \text { IL-6, IL-12, } \\
\text { IL-18 production }\end{array}$} & $(30,35)$ & $(31,34)$ & $(32,33,34,36)$ & $\begin{array}{c}(36) \\
\text { (high doses) }\end{array}$ & $(30,34,36)$ & $\begin{array}{c}(36) \\
\text { (high doses) } \\
\end{array}$ \\
\hline \multicolumn{2}{|c|}{$\begin{array}{l}\text { Promotes OXPHOS and PPP- } \\
\text { driven ROS production }\end{array}$} & $(35,39)$ & $(35,40)$ & & & & \\
\hline \multicolumn{2}{|c|}{$\begin{array}{l}\text { Alkylates KEAP1 and activates } \\
\text { Nrf2 signaling }\end{array}$} & & (34) & \multicolumn{2}{|c|}{$(33,34,41)$} & \multicolumn{2}{|c|}{$(34,40)$} \\
\hline \multicolumn{2}{|c|}{ Reduces IкB levels via ATF3 } & & (34) & \multicolumn{2}{|c|}{ (34) } & \multicolumn{2}{|c|}{$(34,40)$} \\
\hline
\end{tabular}

coordinates the assimilation of a preferred compound over other potential carbon sources (46). CCR in Pseudomonas is significantly different from that in Firmicutes or even in the Enterobacteriaceae. For example, the preferred carbon sources for $P$. aeruginosa are organic acids, particularly succinate, whereas $S$. aureus and E. coli preferentially consume glucose over other carbon sources [reviewed in (46)] (Figure 2). Therefore, in a setting replete with succinate, such as the inflamed airway, $P$. aeruginosa would benefit from the abundant supply of its preferred carbon source.

$P$. aeruginosa grown in a high succinate concentration in vitro induced more inflammation and succinate release in vivo, and exhibited increased colonization of the murine airway (22). However, the continuous assimilation of succinate by $P$. aeruginosa, as is the case in the CF airway, results in increased bacterial production of endogenous ROS via aerobic respiration, providing a steep selective pressure for ROS-adapted isolates. These successful isolates increase metabolic flux through the glyoxylate shunt and the Entner-Doudoroff pathway, bypassing aerobic respiration to produce extracellular polysaccharides (EPS) such as alginate, which are used to produce biofilms and shield the bacteria from oxidant stress (22) (Figure 2). Exogenous ROS from activated phagocytes also contribute to the selection of these hostadapted isolates, which are characterized by decreased LPS at their surface, reduced toxin production, and increased biofilm synthesis $(22,27)$ (Figure 3). Of note, increased EPS surface display by these isolates promotes more itaconate production, creating a positive feedback loop that drives intractable infection $(22,27)$.

Itaconate is toxic to many bacterial species because it inhibits isocitrate lyase (AceA), a key enzyme of the glyoxylate shunt, which is required for bacterial survival in vivo (47-54). $P$. aeruginosa harbors three genes, namely $i c t$, $i c h$ and $c c l$, that enable it to catabolize itaconate and produce the energetic intermediates acetyl-coA and pyruvate $(27,55)$. Clinical isolates from $\mathrm{CF}$ airways prefer to use itaconate over succinate as a carbon source and, unlike the laboratory strain PAO1, are impaired in their ability to infect $\operatorname{Irg} 1^{-/-}$mice (27), exemplifying how in vivo adaptation alters both the metabolic preferences and immunostimulatory capacity of these Gram-negative bacteria. Itaconate thus serves as a key metabolic signal that works in concert with other metabolites, like succinate, to promote $P$. aeruginosa adaptation to the airway.

\section{S. AUREUS INDUCES AN ITACONATE- DOMINATED IMMUNOMETABOLIC RESPONSE}

As a Gram-positive pathogen lacking LPS, S. aureus induces a distinct immunometabolic response from the one detailed above for $P$. aeruginosa. During $S$. aureus infection, itaconate, but not succinate, accumulates in the airway $(22,56)$ (Figure 3). This itaconate-dominated response is accompanied by a distinct cytokine profile, characterized by diminished levels of some pro-inflammatory cytokines, like IL-1 $\beta$ and IL-6, but not others, like TNF $\alpha$ (56). The selective reduction in IL-1 $\beta$ and IL-6 is likely due to the inability of $S$. aureus to stimulate TLR4driven succinate accumulation and HIF- $1 \alpha$ stabilization.

The host immune response to $S$. aureus infection depends on TLR2, which responds to bacterial cell surface and secreted factors like lipopeptides (57). TLR2 plays a prominent role in the immune response to early staphylococcal colonization of the 


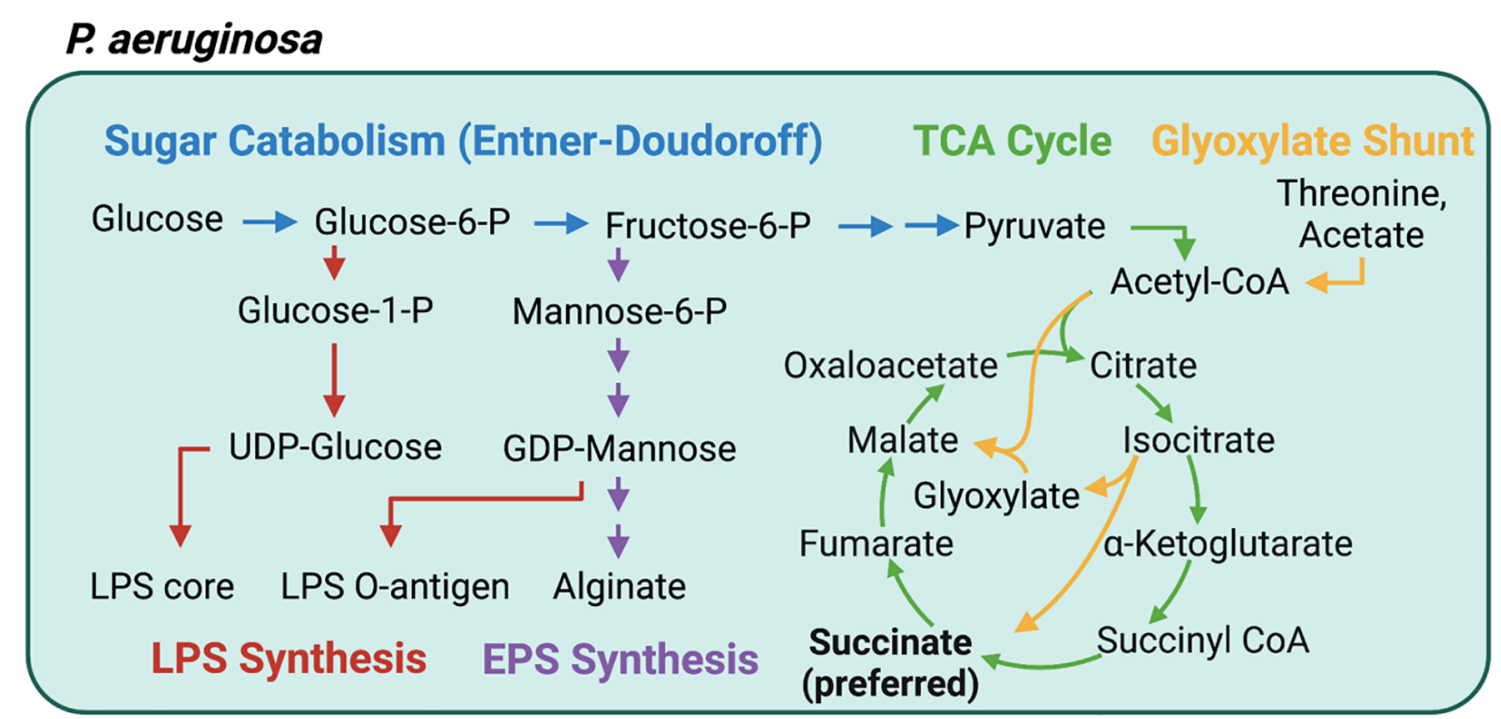

\section{S. aureus}

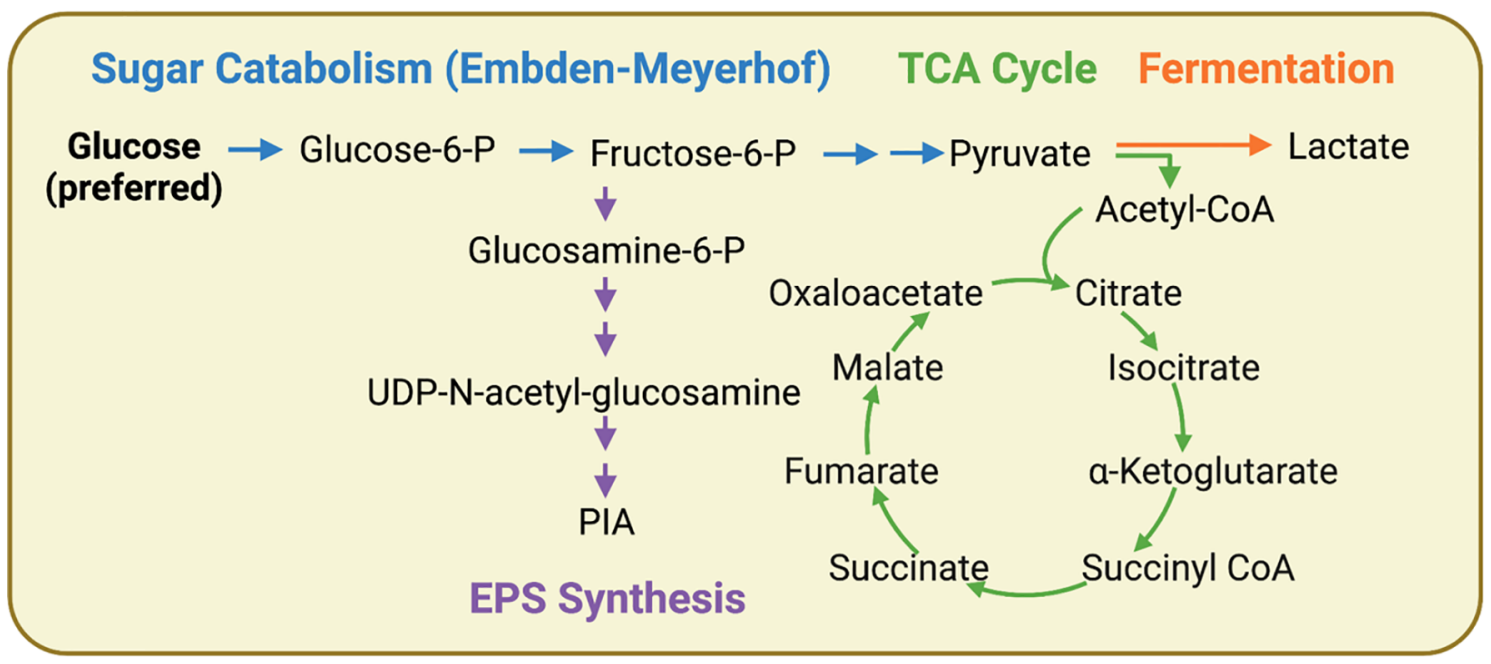

FIGURE 2 | Interconnected pathways contributing to central carbon metabolism, LPS, and EPS synthesis in P. aeruginosa and S. aureus. P. aeruginosa and S. aureus have distinct carbon preferences and metabolic pathways that contribute to pathogenesis. Carbon catabolite repression (CCR) ensures that $P$. aeruginosa consumes succinate until it is depleted whereas $S$. aureus preferentially consumes glucose. The continuous consumption of succinate by $P$. aeruginosa generates endogenous bacterial reactive oxygen species (ROS) via increased aerobic respiration. $P$. aeruginosa adapts by bypassing OXPHOS and upregulating the glyoxylate shunt and the Entner-Doudoroff pathways, resulting in increased extracellular polysaccharide (EPS) synthesis. S. aureus, meanwhile, is highly dependent on glycolysis and fermentative metabolism for survival during infection. When glycolysis is interrupted, as it is in itaconate-rich environments, carbon is shunted into EPS synthesis.

lung, but is not essential for bacterial clearance and host survival during pneumonia (58), suggesting that there are other pathways that the host uses to sense and respond to $S$. aureus during lung infection. This redundancy is important, given that $S$. aureus can produce super antigens that bind and inactivate TLR2 (59).

Though IL-1 $\beta$ and IL-6 are less abundant in the S. aureusinfected airway, they still play important roles in infection outcomes. As in $P$. aeruginosa lung infections, IL-1 $\beta$ does not facilitate bacterial clearance and instead exacerbates tissue damage during $S$. aureus pneumonia. Mice lacking the interferon receptor Ifnlr1 demonstrate reduced IL-1 $\beta$ production along with improved bacterial clearance during $S$. aureus lung infection, and administration of recombinant IL-1 $\beta$ to these mice worsens bacterial burden (60). During $S$. aureus lung infections, IL-1 $\beta$ is generated via inflammasome-dependent and independent mechanisms, including neutrophil elastase (60). These alternative mechanisms likely sustain IL- $1 \beta$ production during chronic staphylococcal infections, given that inflammasome-driven inflammation and tissue damage is dependent on S. aureus alpha toxin (Hla), which is often downregulated in clinical strains $(56,61)$. IL-6, meanwhile, modulates lung inflammation through mechanisms that depend on the nature of the stimulus and the 
severity of inflammation, promoting neutrophil infiltration in response to staphylococcal peptidoglycan but limiting neutrophil infiltration, cytokine production, and tissue damage in response to staphylococcal lipoteichoic acid (62).

The role of metabolites in regulating inflammation during $S$. aureus lung infection has not yet been defined. The majority of the studies that delineated the interplay between inflammation and metabolism used LPS as a stimulus, and thus involved TLR4related pathways that may not be relevant to $S$. aureus infection. Given that LPS is often used to stimulate immunometabolic reprogramming, another major question that remains is how $S$. aureus induces itaconate production. A recent study of the role of itaconate in $S$. aureus endophthalmitis indicates that TLR2 signaling is not sufficient, as heat-killed S. aureus, lipoteichoic acid, and peptidoglycan do not stimulate IRG1 expression to the same extent as live bacteria (63). Instead, IRG1 expression can be mitigated by administration of a mitochondrial ROS scavenger, suggesting that host mitochondrial stress and/or oxidants promote IRG1 expression during S. aureus infection (56).
Mitochondrial ROS production and itaconate accumulation in the murine airway depend on S. aureus glycolysis, demonstrating that bacterial metabolism itself stimulates itaconate generation during $S$. aureus lung infection (56). The exact mechanism that connects bacterial metabolism to host metabolic reprogramming still needs to be determined.

\section{ITACONATE DRIVES PERSISTENT S. AUREUS LUNG INFECTION}

Just as $S$. aureus stimulates a distinct immunometabolic response when compared with $P$. aeruginosa, it also employs different strategies for adaptation to the human airway. As mentioned above, $S$. aureus preferentially consumes glucose, and its glucose metabolism is intricately linked with toxin and extracellular polysaccharide synthesis through carbon catabolite repression, regulated by CcpA and CodY [reviewed in (64)]. Increased glucose consumption can also be used to fuel lactate fermentation, which
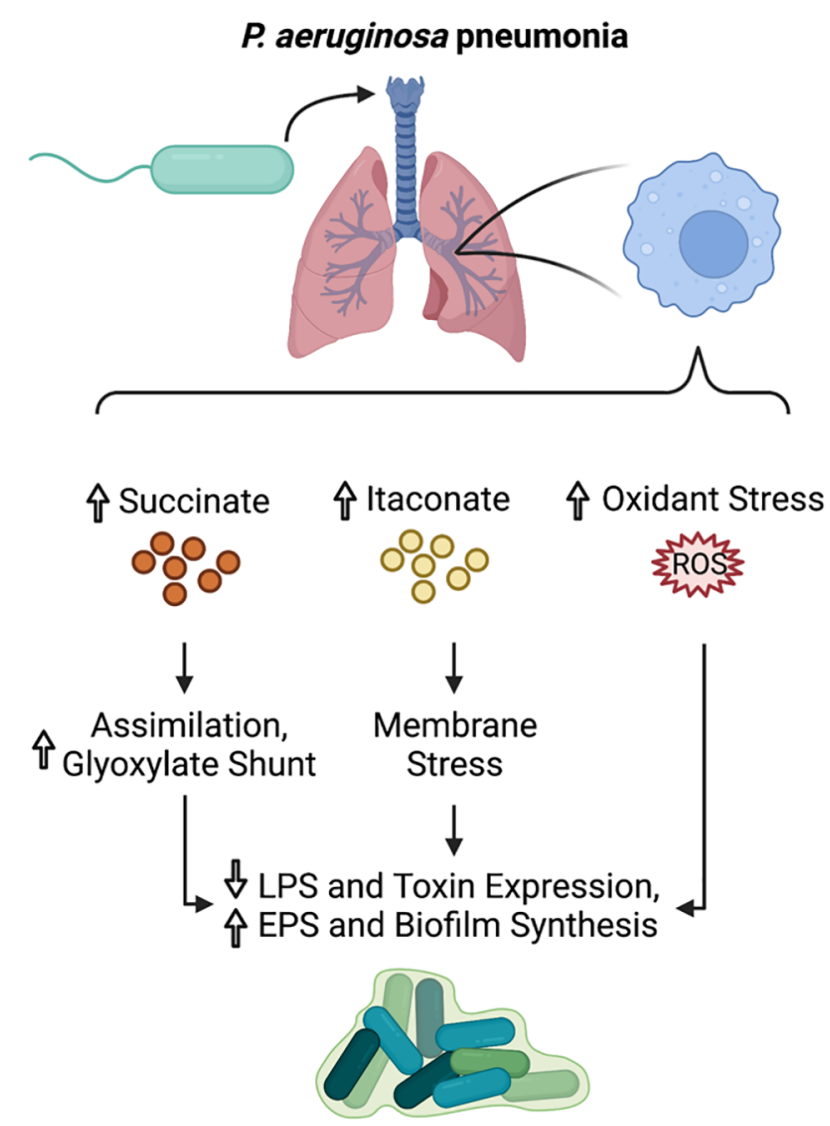

Host-adapted $P$. aeruginosa

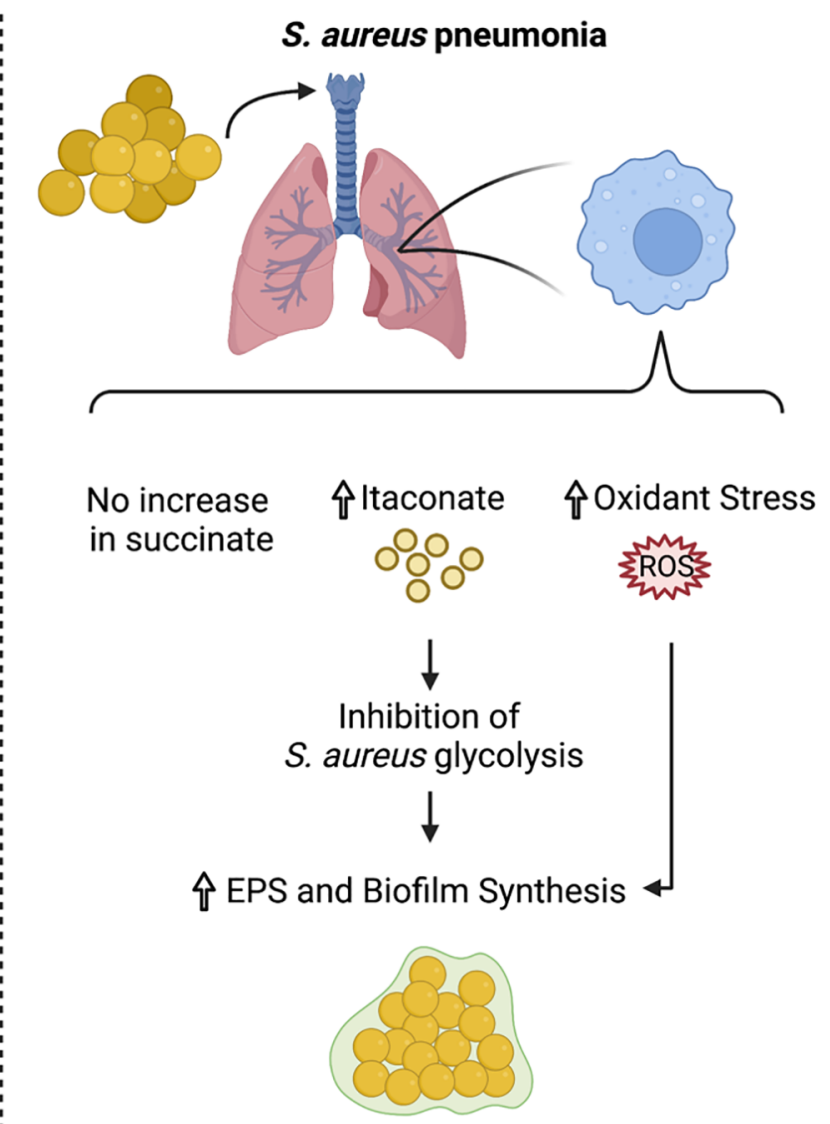

Host-adapted S. aureus

FIGURE 3 | Impact of immunometabolites on $P$. aeruginosa and $S$. aureus adaptation to the lung microenvironment. During infection with $P$. aeruginosa, airway immune cells produce succinate, itaconate, and ROS. This creates a strong selective pressure for isolates that adapt via metabolic rewiring, decreased LPS surface display and toxin production, and increased biofilm synthesis. In contrast, S. aureus infection induces accumulation of itaconate and ROS, but not succinate in the airway. Both itaconate and ROS select for metabolically-altered S. aureus strains that exhibit decreased glycolytic activity and increased EPS and biofilm production. 
enables the bacteria to maintain redox balance within the cell in the setting of oxidant stress (65). As such, glucose consumption is critical for survival during inflammation, and mutants that are unable to transport glucose into the cell or metabolize it via glycolysis are impaired in skin, soft tissue, and lung infections (56, 65-67).

Unlike $P$. aeruginosa, $S$. aureus is unable to catabolize itaconate, and itaconate inhibits $S$. aureus growth in activated host immune cells (53). Instead of targeting the glyoxylate shunt, which is absent in $S$. aureus, itaconate inhibits staphylococcal glycolysis (EmbdenMeyerhof), mirroring one of its mechanisms of action in mammalian cells (56). This glycolytic inhibition rewires bacterial metabolism to promote carbon flux through upstream pathways that synthesize extracellular polysaccharides used in biofilms (56) (Figure 2). As such, longitudinal isolates that represent adaption to the CF lung over 15 years exhibited increased biofilm production in the presence of itaconate (56) (Figure 3). This differs from the mechanism by which itaconate promotes $P$. aeruginosa biofilms, which involves inducing membrane stress to downregulate LPS production and promote EPS synthesis (27). Nevertheless, these adaptive mechanisms converge on biofilm formation, which is beneficial not just to the microorganism as protection from phagocytosis and antimicrobial factors, but also to the bacterial community, which includes both $S$. aureus and P. aeruginosa during chronic lung infections.

While $S$. aureus does not induce succinate accumulation, it is often in succinate rich environments, particularly during coinfection with $P$. aeruginosa in the CF airway. In contrast to its effects on $P$. aeruginosa, succinate inhibits $S$. aureus growth and biofilm production in a dose-dependent manner, likely by inhibiting consumption of its preferred carbon sources, including glucose (22). Accordingly, S. aureus cultured in high succinate concentrations is impaired in its ability to colonize the airway and lungs of mice, unlike $P$. aeruginosa grown in the presence of high succinate concentrations (22). These studies reaffirm that common airway pathogens have different metabolic preferences that are tailored to the immunometabolic response they induce in the host.

\section{CONCLUDING REMARKS}

In recent years, it has become increasingly apparent that immunometabolism plays a key role in the pathogenesis of infections. Microbial metabolism has often been neglected in these studies, with many research groups instead turning to the use of LPS as a standard proxy for bacterial stimulation. There is increasing evidence, however, that microbial metabolic flexibility is not only critical for bacterial persistence during infection, but also in shaping the host immunometabolic response (68-70). Importantly, this metabolic interplay between host and pathogen varies by microbe and infection site. Future investigations into the immunometabolism of infection should continue to include diverse pathogens and tissues, taking care to address the differences in host response to active microbial metabolism versus inert PAMPs. These studies should also address the systemic consequences of immunometabolism, as comparatively little is known about the activity of metabolites that are absorbed into the bloodstream during infection.
While we have focused on the roles of just a few metabolites in driving bacterial adaptation, other airway metabolites produced during infection are likely to influence host-pathogen metabolic interactions and infection outcomes. For example, analysis of sputum samples from $\mathrm{CF}$ and non-CF patients revealed higher amino acid concentrations in CF sputum (71). This is particularly interesting given that both $P$. aeruginosa and $S$. aureus consume amino acids, with a hierarchical preference for some amino acids over others (72) [reviewed in (73)]. Conversely, amino acid starvation in many pathogenic bacteria induces virulence gene expression. Thus, it is tempting to speculate that the abundance of amino acids in the CF airway progressively leads to decreased virulence gene expression as a result of increased amino acid consumption.

Other factors that may influence the metabolic interaction between the host and pathogen include the contribution of microbiota-derived metabolites or metabolites generated by coinfecting pathogens. Microbiome-derived metabolites such as short chain fatty acids have been shown to regulate the function of key immune cells, including $\mathrm{CD}^{+} \mathrm{T}$ cells and their ability to recall infection (74). During co-infection of the CF lung, $S$. aureus produces acetoin, which is used as a carbon source by $P$. aeruginosa, decreasing the toxic effect of accumulated acetoin on $S$. aureus and promoting the persistence of both bacteria (75). These studies highlight the importance of considering the metabolites originating from organisms other than the host and pathogen of interest when studying the metabolic underpinnings of infection pathogenesis.

Microbial adaptation to the host can be difficult to study because animal models of chronic pulmonary infection are limited. This problem is often circumvented by using clinical isolates that have adapted to the human airway. One caveat to this approach is that these metabolic adaptations may not be maintained over time as the isolates are repeatedly exposed to artificial in vitro conditions that do not adequately mimic the tissue microenvironment. Altogether, while targeting host immunometabolism as an alternative or complementary therapeutic strategy to enhance the clearance of multidrug-resistant bacteria is an alluring prospect, host-pathogen metabolic interactions and additional factors that influence these dynamics require further study.

\section{AUTHOR CONTRIBUTIONS}

KLT, ASP, and TWFL wrote and edited the manuscript. All authors have read and agreed to the published version of the manuscript.

\section{FUNDING}

ASP was supported by NIH grant 1R35HL 135800 and the Cystic Fibrosis Foundation CFF PRINCE18G0.

\section{ACKNOWLEDGMENTS}

Figures were created with Biorender.com. 


\section{REFERENCES}

1. Galeas-Pena M, McLaughlin N, Pociask D. The Role of the Innate Immune System on Pulmonary Infections. Biol Chem (2019) 400(4):443-56. doi: 10.1515/hsz-2018-0304

2. Iwasaki A, Foxman EF, Molony RD. Early Local Immune Defences in the Respiratory Tract. Nat Rev Immunol (2017) 17(1):7-20. doi: 10.1038/nri.2016.117

3. Holguin F. Oxidative Stress in Airway Diseases. Ann Am Thorac Soc (2013) 10 Suppl:S150-7. doi: 10.1513/AnnalsATS.201305-116AW

4. Bianconi I, Milani A, Cigana C, Paroni M, Levesque RC, Bertoni G, et al. Positive Signature-Tagged Mutagenesis in Pseudomonas Aeruginosa: Tracking Patho-Adaptive Mutations Promoting Airways Chronic Infection. PLoS Pathogens (2011) 7(2):e1001270. doi: 10.1371/journal.ppat.1001270

5. Blattner S, Das S, Paprotka K, Eilers U, Krischke M, Kretschmer D, et al. Staphylococcus Aureus Exploits a Non-Ribosomal Cyclic Dipeptide to Modulate Survival Within Epithelial Cells and Phagocytes. PLoS Pathogens (2016) 12(9):e1005857. doi: 10.1371/journal.ppat.1005857

6. Gabryszewski SJ, Wong Fok Lung T, Annavajhala MK, Tomlinson KL, Riquelme SA, Khan IN, et al. Metabolic Adaptation in Methicillin-Resistant Staphylococcus Aureus Pneumonia. Am J Respir Cell Mol Biol (2019) 61 (2):185-97. doi: 10.1165/rcmb.2018-0389OC

7. Tan X, Coureuil M, Ramond E, Euphrasie D, Dupuis M, Tros F, et al. Chronic Staphylococcus Aureus Lung Infection Correlates With Proteogenomic and Metabolic Adaptations Leading to an Increased Intracellular Persistence. Clin Infect Dis (2019) 69(11):1937-45. doi: 10.1093/cid/ciz106

8. Bernardy EE, Petit RA3rd, Raghuram V, Alexander AM, Read TD, Goldberg JB. Genotypic and Phenotypic Diversity of Staphylococcus Aureus Isolates From Cystic Fibrosis Patient Lung Infections and Their Interactions With Pseudomonas Aeruginosa. mBio (2020) 11(3):e00735-20. doi: 10.1128/mBio.00735-20

9. Gaspar MC, Couet W, Olivier JC, Pais AA, Sousa JJ. Pseudomonas Aeruginosa Infection in Cystic Fibrosis Lung Disease and New Perspectives of Treatment: A Review. Eur J Clin Microbiol Infect Dis (2013) 32(10):1231-52. doi: 10.1007/s10096-013-1876-y

10. Hubert D, Reglier-Poupet H, Sermet-Gaudelus I, Ferroni A, Le Bourgeois M, Burgel PR, et al. Association Between Staphylococcus Aureus Alone or Combined With Pseudomonas Aeruginosa and the Clinical Condition of Patients With Cystic Fibrosis. J Cyst Fibros (2013) 12(5):497-503. doi: 10.1016/j.jcf.2012.12.003

11. Fischer AJ, Singh SB, LaMarche MM, Maakestad LJ, Kienenberger ZE, Pena TA, et al. Sustained Coinfections With Staphylococcus Aureus and Pseudomonas Aeruginosa in Cystic Fibrosis. Am J Respir Crit Care Med (2021) 203(3):328-38. doi: 10.1164/rccm.202004-1322OC

12. Raoust E, Balloy V, Garcia-Verdugo I, Touqui L, Ramphal R, Chignard M. Pseudomonas Aeruginosa LPS or Flagellin Are Sufficient to Activate TLRDependent Signaling in Murine Alveolar Macrophages and Airway Epithelial Cells. PLoS One (2009) 4(10):e7259. doi: 10.1371/journal.pone.0007259

13. Feuillet V, Medjane S, Mondor I, Demaria O, Pagni PP, Galan JE, et al. Involvement of Toll-Like Receptor 5 in the Recognition of Flagellated Bacteria. Proc Natl Acad Sci U S A (2006) 103(33):12487-92. doi: 10.1073/pnas.0605200103

14. Ramphal R, Balloy V, Jyot J, Verma A, Si-Tahar M, Chignard M. Control of Pseudomonas Aeruginosa in the Lung Requires the Recognition of Either Lipopolysaccharide or Flagellin. J Immunol (2008) 181(1):586-92. doi: 10.4049/jimmunol.181.1.586

15. Kelly B, O'Neill LA. Metabolic Reprogramming in Macrophages and Dendritic Cells in Innate Immunity. Cell Res (2015) 25(7):771-84. doi: 10.1038/cr.2015.68

16. Warburg O, Wind F, Negelein E. The Metabolism of Tumors in the Body. J Gen Physiol (1927) 8(6):519-30. doi: 10.1085/jgp.8.6.519

17. Tannahill GM, Curtis AM, Adamik J, Palsson-McDermott EM, McGettrick AF, Goel G, et al. Succinate Is an Inflammatory Signal That Induces IL-1beta Through HIF-1alpha. Nature (2013) 496(7444):238-42. doi: 10.1038/nature11986

18. Mills EL, Kelly B, Logan A, Costa ASH, Varma M, Bryant CE, et al. Succinate Dehydrogenase Supports Metabolic Repurposing of Mitochondria to Drive Inflammatory Macrophages. Cell (2016) 167(2):457-70.e13. doi: 10.1016/ j.cell.2016.08.064

19. Selak MA, Armour SM, MacKenzie ED, Boulahbel H, Watson DG, Mansfield $\mathrm{KD}$, et al. Succinate Links TCA Cycle Dysfunction to Oncogenesis by Inhibiting HIF-Alpha Prolyl Hydroxylase. Cancer Cell (2005) 7(1):77-85. doi: 10.1016/ j.ccr.2004.11.022

20. Zasłona Z, O’Neill LAJ. Cytokine-Like Roles for Metabolites in Immunity. Mol Cell (2020) 78(5):814-23. doi: 10.1016/j.molcel.2020.04.002
21. Liu P-S, Wang H, Li X, Chao T, Teav T, Christen S, et al. $\alpha$-Ketoglutarate Orchestrates Macrophage Activation Through Metabolic and Epigenetic Reprogramming. Nat Immunol (2017) 18(9):985-94. doi: 10.1038/ni.3796

22. Riquelme SA, Lozano C, Moustafa AM, Liimatta K, Tomlinson KL, Britto C, et al. CFTR-PTEN-Dependent Mitochondrial Metabolic Dysfunction Promotes Pseudomonas Aeruginosa Airway Infection. Science Translational Medicine. (2019) 11(499):aav4634. doi: 10.1126/scitranslmed.aav4634

23. Riquelme SA, Hopkins BD, Wolfe AL, DiMango E, Kitur K, Parsons R, et al. Cystic Fibrosis Transmembrane Conductance Regulator Attaches Tumor Suppressor PTEN to the Membrane and Promotes Anti. Pseudomonas aeruginosa Immunity Immunity (2017) 47(6):1169-81.e7. doi: 10.1016/ j.immuni.2017.11.010

24. Ragab D, Salah Eldin H, Taeimah M, Khattab R, Salem R. The COVID-19 Cytokine Storm; What We Know So Far. Front Immunol (2020) 11:1446. doi: 10.3389/fimmu.2020.01446

25. Hojyo S, Uchida M, Tanaka K, Hasebe R, Tanaka Y, Murakami M, et al. How COVID-19 Induces Cytokine Storm With High Mortality. Inflamm Regen (2020) 40:37. doi: 10.1186/s41232-020-00146-3

26. Cohen TS, Prince AS. Activation of Inflammasome Signaling Mediates Pathology of Acute P. Aeruginosa Pneumonia. J Clin Invest (2013) 123 (4):1630-7. doi: 10.1172/JCI66142

27. Riquelme SA, Liimatta K, Wong Fok Lung T, Fields B, Ahn D, Chen D, et al. Pseudomonas Aeruginosa Utilizes Host-Derived Itaconate to Redirect Its Metabolism to Promote Biofilm Formation. Cell Metab (2020) 31(6):1091106.e6. doi: 10.1016/j.cmet.2020.04.017

28. Baup S. Ueber Eine Neue Brenz - Citronensäure Und Über Die Nomenclatur Der Brenzkörper Überhaupt. J Für Praktische Chem (2004) 8(1):418-24. doi: $10.1002 /$ prac. 18360080162

29. Cordes T, Wallace M, Michelucci A, Divakaruni AS, Sapcariu SC, Sousa C, et al. Immunoresponsive Gene 1 and Itaconate Inhibit Succinate Dehydrogenase to Modulate Intracellular Succinate Levels. J Biol Chem (2016) 291(27):14274-84. doi: 10.1074/jbc.M115.685792

30. Lampropoulou V, Sergushichev A, Bambouskova M, Nair S, Emma, Loginicheva E, et al. Itaconate Links Inhibition of Succinate Dehydrogenase With Macrophage Metabolic Remodeling and Regulation of Inflammation. Cell Metab (2016) 24(1):158-66. doi: 10.1016/j.cmet.2016.06.004

31. Qin W, Qin K, Zhang Y, Jia W, Chen Y, Cheng B, et al. S-Glycosylation-Based Cysteine Profiling Reveals Regulation of Glycolysis by Itaconate. Nat Chem Biol (2019) 15(10):983-91. doi: 10.1038/s41589-019-0323-5

32. Liao S-T, Han C, Xu D-Q, Fu X-W, Wang J-S, Kong L-Y. 4-Octyl Itaconate Inhibits Aerobic Glycolysis by Targeting GAPDH to Exert Anti-Inflammatory Effects. Nat Commun (2019) 10(1):5091. doi: 10.1038/s41467-019-13078-5

33. Mills EL, Ryan DG, Prag HA, Dikovskaya D, Menon D, Zaslona Z, et al. Itaconate Is an Anti-Inflammatory Metabolite That Activates Nrf2 via alkylation of KEAP1. Nature (2018) 556(7699):113-7. doi: 10.1038/nature25986

34. Swain A, Bambouskova M, Kim H, Andhey PS, Duncan D, Auclair K, et al. Comparative Evaluation of Itaconate and its Derivatives Reveals Divergent Inflammasome and Type I Interferon Regulation in Macrophages. Nat Metab (2020) 2:594-602. doi: 10.1038/s42255-020-0210-0

35. Zhu X, Guo Y, Liu Z, Yang J, Tang H, Wang Y. Itaconic Acid Exerts AntiInflammatory and Antibacterial Effects via Promoting Pentose Phosphate Pathway to Produce ROS. Sci Rep (2021) 11(1):18173. doi: 10.1038/s41598-021-97352-x

36. Muri J, Wolleb H, Broz P, Carreira EM, Kopf M. Electrophilic Nrf2 Activators and Itaconate Inhibit Inflammation at Low Dose and Promote IL-1 $\beta$ Production and Inflammatory Apoptosis at High Dose. Redox Biol (2020) 36:101647. doi: 10.1016/j.redox.2020.101647

37. Bambouskova M, Potuckova L, Paulenda T, Kerndl M, Mogilenko DA, Lizotte $\mathrm{K}$, et al. Itaconate Confers Tolerance to Late NLRP3 Inflammasome Activation. Cell Rep (2021) 34(10):108756. doi: 10.1016/j.celrep.2021.108756

38. Hooftman A, Angiari S, Hester S, Corcoran SE, Runtsch MC, Ling C, et al. The Immunomodulatory Metabolite Itaconate Modifies NLRP3 and Inhibits Inflammasome Activation. Cell Metab (2020) 32(3):468-78.e7. doi: 10.1016/ j.cmet.2020.07.016

39. Weiss JM, Davies LC, Karwan M, Ileva L, Ozaki MK, Cheng RYS, et al. Itaconic Acid Mediates Crosstalk Between Macrophage Metabolism and Peritoneal Tumors. J Clin Invest (2018) 128(9):3794-805. doi: 10.1172/JCI99169

40. Bambouskova M, Gorvel L, Lampropoulou V, Sergushichev A, Loginicheva E, Johnson K, et al. Electrophilic Properties of Itaconate and Derivatives Regulate 
the Iкb $\zeta-A T F 3$ Inflammatory Axis. Nature (2018) 556(7702):501-4. doi: 10.1038/s41586-018-0052-z

41. Liu H, Feng Y, Xu M, Yang J, Wang Z, Di G. Four-Octyl Itaconate Activates Keap1-Nrf2 Signaling to Protect Neuronal Cells From Hydrogen Peroxide. Cell Commun Signaling (2018) 16(1):81. doi: 10.1186/s12964-018-0294-2

42. Faas M, Ipseiz N, Ackermann J, Culemann S, Gruneboom A, Schroder F, et al. IL-33-Induced Metabolic Reprogramming Controls the Differentiation of Alternatively Activated Macrophages and the Resolution of Inflammation. Immunity (2021) 54(11):2531-46.e5. doi: 10.1016/j.immuni.2021.09.010

43. O'Carroll SM, O'Neill LAJ. Targeting Immunometabolism to Treat COVID19. Immunother $A d v$ (2021) 1(1):Itab013. doi: 10.1093/immadv/ltab013

44. Daniels BP, Kofman SB, Smith JR, Norris GT, Snyder AG, Kolb JP, et al. The Nucleotide Sensor ZBP1 and Kinase RIPK3 Induce the Enzyme IRG1 to Promote an Antiviral Metabolic State in Neurons. Immunity (2019) 50(1):6476 e4. doi: 10.1016/j.immuni.2018.11.017

45. Sohail A, Iqbal AA, Sahini N, Tantawy M, Winterhoff M, Ebensen T, et al. Itaconate and Derivatives Reduce Interferon Response and Inflammation in Influenza A Virus Infection. bioRxiv (2021) 2021.01.20.427392. doi: 10.1101/ 2021.01.20.427392

46. Rojo F. Carbon Catabolite Repression in Pseudomonas : Optimizing Metabolic Versatility and Interactions With the Environment. FEMS Microbiol Rev (2010) 34(5):658-84. doi: 10.1111/j.1574-6976.2010.00218.x

47. Williams JO, Roche TE, McFadden BA. Mechanism of Action of Isocitrate Lyase From Pseudomonas Indigofera. Biochemistry (1971) 10(8):1384-90. doi: 10.1021/bi00784a017

48. Lorenz MC, Fink GR. The Glyoxylate Cycle is Required for Fungal Virulence. Nature (2001) 412(6842):83-6. doi: 10.1038/35083594

49. McFadden BA, Purohit S. Itaconate, an Isocitrate Lyase-Directed Inhibitor in Pseudomonas Indigofera. J Bacteriol (1977) 131(1):136-44. doi: 10.1128/ jb.131.1.136-144.1977

50. McKinney JD, Honer zu Bentrup K, Munoz-Elias EJ, Miczak A, Chen B, Chan WT, et al. Persistence of Mycobacterium Tuberculosis in Macrophages and Mice Requires the Glyoxylate Shunt Enzyme Isocitrate Lyase. Nature (2000) 406(6797):735-8. doi: 10.1038/35021074

51. Michelucci A, Cordes T, Ghelfi J, Pailot A, Reiling N, Goldmann O, et al. Immune-Responsive Gene 1 Protein Links Metabolism to Immunity by Catalyzing Itaconic Acid Production. Proc Natl Acad Sci U S A (2013) 110 (19):7820-5. doi: 10.1073/pnas.1218599110

52. Munoz-Elias EJ, McKinney JD. Mycobacterium Tuberculosis Isocitrate Lyases 1 and 2 are Jointly Required for. Vivo Growth Virulence Nat Med (2005) 11(6):638-44. doi: 10.1038/nm1252

53. Naujoks J, Tabeling C, Dill BD, Hoffmann C, Brown AS, Kunze M, et al. IFNs Modify the Proteome of Legionella-Containing Vacuoles and Restrict Infection Via IRG1-Derived Itaconic Acid. PLoS Pathogens (2016) 12(2): e1005408. doi: 10.1371/journal.ppat.1005408

54. Lacey CA, Ponzilacqua-Silva B, Chambers CA, Dadelahi AS, Skyberg JA, Palmer GH. MyD88-Dependent Glucose Restriction and Itaconate Production Control Brucella Infection. Infect Immunity (2021) 89(10): e00156-21. doi: 10.1128/IAI.00156-21

55. Sasikaran J, Ziemski M, Zadora PK, Fleig A, Berg IA. Bacterial Itaconate Degradation Promotes Pathogenicity. Nat Chem Biol (2014) 10(5):371-7. doi: $10.1038 /$ nchembio. 1482

56. Kira L, Tomlinson TWFL, Dach F, Medini KA, Stanislaw JG, . Groves RA, et al. Staphylococcus Aureus Induces an Itaconate-Dominated Immunometabolic Response That Drives Biofilm Formation. Nat Commun (2021) 12:1399. doi: 10.1038/s41467-021-21718-y

57. Hanzelmann D, Joo H-S, Franz-Wachtel M, Hertlein T, Stevanovic S, Macek B, et al. Toll-Like Receptor 2 Activation Depends on Lipopeptide Shedding by Bacterial Surfactants. Nat Commun (2016) 7(1):12304. doi: 10.1038/ ncomms 12304

58. Skerrett SJ, Braff MH, Liggitt HD, Rubens CE. Toll-Like Receptor 2 has a Prominent But Nonessential Role in Innate Immunity to Staphylococcus Aureus Pneumonia. Physiol Rep (2017) 5(21):e13491. doi: 10.14814/ phy2.13491

59. Koymans KJ, Feitsma LJ, Brondijk THC, Aerts PC, Lukkien E, Lössl P, et al. Structural Basis for Inhibition of TLR2 by Staphylococcal Superantigen-Like Protein 3 (SSL3). Proc Natl Acad Sci (2015) 112(35):11018-23. doi: 10.1073/ pnas. 1502026112
60. Pires S, Parker D. IL-1 $\beta$ Activation in Response to Staphylococcus Aureus Lung Infection Requires Inflammasome-Dependent and Independent Mechanisms. Eur J Immunol (2018) 48(10):1707-16. doi: 10.1002/ eji.201847556

61. Kebaier C, Chamberland RR, Allen IC, Gao X, Broglie PM, Hall JD, et al. Staphylococcus Aureus $\alpha$-Hemolysin Mediates Virulence in a Murine Model of Severe Pneumonia Through Activation of the NLRP3 Inflammasome. J Infect Dis (2012) 205(5):807-17. doi: 10.1093/infdis/jir846

62. Leemans JC, Vervoordeldonk MJBM, Florquin S, Van Kessel KP, van der Poll T. Differential Role of Interleukin-6 in Lung Inflammation Induced by Lipoteichoic Acid and Peptidoglycan Fromstaphylococcus Aureus. Am J Respir Crit Care Med (2002) 165(10):1445-50. doi: 10.1164/rccm.2106045

63. Singh S, Singh PK, Jha A, Naik P, Joseph J, Giri S, et al. Integrative Metabolomics and Transcriptomics Identifies Itaconate as an Adjunct Therapy to Treat Ocular Bacterial Infection. Cell Rep Med (2021) 2 (5):100277. doi: 10.1016/j.xcrm.2021.100277

64. Somerville GA, Proctor RA. At the Crossroads of Bacterial Metabolism and Virulence Factor Synthesis in Staphylococci. Microbiol Mol Biol Rev (2009) 73 (2):233-48. doi: 10.1128/MMBR.00005-09

65. Vitko NP, Spahich NA, Richardson AR. Glycolytic Dependency of High-Level Nitric Oxide Resistance and Virulence in Staphylococcus aureus. mBio (2015) 6(2):e00045-15. doi: 10.1128/mBio.00045-15

66. Wickersham M, Wachtel S, Wong Fok Lung T, Soong G, Jacquet R, Richardson A, et al. Metabolic Stress Drives Keratinocyte Defenses Against Staphylococcus aureus Infection. Cell Rep (2017) 18(11):2742-51. doi: 10.1016/j.celrep.2017.02.055

67. Wong Fok Lung T, Monk IR, Acker KP, Mu A, Wang N, Riquelme SA, et al. Staphylococcus Aureus Small Colony Variants Impair Host Immunity by Activating Host Cell Glycolysis and Inducing Necroptosis. Nat Microbiol (2020) 5(1):141-53. doi: 10.1038/s41564-019-0597-0

68. Heim CE, Bosch ME, Yamada KJ, Aldrich AL, Chaudhari SS, Klinkebiel D, et al. Lactate Production by Staphylococcus Aureus Biofilm Inhibits HDAC11 to Reprogramme the Host Immune Response During Persistent Infection. Nat Microbiol (2020) 5(10):1271-84. doi: 10.1038/s41564-020-0756-3

69. Chen X, Teoh WP, Stock MR, Resko ZJ, Alonzo F. Branched Chain Fatty Acid Synthesis Drives Tissue-Specific Innate Immune Response and Infection Dynamics of Staphylococcus Aureus. PLoS Pathogens (2021) 17(9): e1009930. doi: 10.1371/journal.ppat.1009930

70. Teoh WP, Chen X, Laczkovich I, Alonzo F. Staphylococcus Aureus Adapts to the Host Nutritional Landscape to Overcome Tissue-Specific Branched-Chain Fatty Acid Requirement. Proc Natl Acad Sci (2021) 118(13):e2022720118. doi: $10.1073 /$ pnas.2022720118

71. Schwab U, Abdullah LH, Perlmutt OS, Albert D, Davis CW, Arnold RR, et al. Localization of Burkholderia Cepacia Complex Bacteria in Cystic Fibrosis Lungs and Interactions With Pseudomonas Aeruginosa in Hypoxic Mucus. Infect Immunity (2014) 82(11):4729-45. doi: 10.1128/IAI.01876-14

72. Halsey CR, Lei S, Wax JK, Lehman MK, Nuxoll AS, Steinke L, et al. Amino Acid Catabolism in Staphylococcus Aureus and the Function of Carbon Catabolite Repression. mBio (2017) 8(1):e01434-16. doi: 10.1128/mBio.01434-16

73. La R. Johansen, and Molin. Adapting to the Airways: Metabolic Requirements of Pseudomonas Aeruginosa During the Infection of Cystic Fibrosis Patients. Metabolites (2019) 9(10):234. doi: 10.3390/metabo9100234

74. Bachem A, Makhlouf C, Binger KJ, de Souza DP, Tull D, Hochheiser K, et al. Microbiota-Derived Short-Chain Fatty Acids Promote the Memory Potential of Antigen-Activated CD8(+) T Cells. Immunity (2019) 51(2):285-97.e5. doi: 10.1016/j.immuni.2019.06.002

75. Camus L, Briaud P, Bastien S, Elsen S, Doleans-Jordheim A, Vandenesch F, et al. Trophic Cooperation Promotes Bacterial Survival of Staphylococcus Aureus and Pseudomonas Aeruginosa. ISME J (2020) 14(12):3093-105. doi: 10.1038/s41396-020-00741-9

Conflict of Interest: The authors declare that the research was conducted in the absence of any commercial or financial relationships that could be construed as a potential conflict of interest.

Publisher's Note: All claims expressed in this article are solely those of the authors and do not necessarily represent those of their affiliated organizations, or those of the publisher, the editors and the reviewers. Any product that may be evaluated in 
this article, or claim that may be made by its manufacturer, is not guaranteed or endorsed by the publisher.

Copyright $\odot 2021$ Tomlinson, Prince and Wong Fok Lung. This is an open-access article distributed under the terms of the Creative Commons Attribution License
(CC BY). The use, distribution or reproduction in other forums is permitted, provided the original author(s) and the copyright owner(s) are credited and that the original publication in this journal is cited, in accordance with accepted academic practice. No use, distribution or reproduction is permitted which does not comply with these terms. 